\title{
CAVITATION EROSION DAMAGE OF SCROLL STEEL PLATES BY HIGH-SPEED GAS WORKING FLUID
}

\author{
I. TZANAKIS ${ }^{1,3}$, A. GEORGOULAS ${ }^{2,4}$, M. HADFIELD $^{3} \&$ N. KOTSOVINOS ${ }^{4}$ \\ ${ }^{1}$ Brunel Center for Advanced Solidification Technology (BCAST), Brunel University, \\ Uxbridge, Middlesex UB8 3PH. \\ ${ }^{2}$ Department of Engineering, Thermal Physics Group, University of Bergamo, \\ Viale Marconi 5, 24044 Dalmine, Italy. \\ ${ }^{3}$ Sustainable Design Research Centre, School of Design, Engineering and Computing, \\ Bournemouth University, UK. \\ ${ }^{4}$ Laboratory of Hydraulics and Hydraulic Structures, Fluid Mechanics Division, \\ Department of Civil Engineering, Democritus University of Thrace, Greece.
}

\begin{abstract}
A steel plate is one of the critical components of a scroll expander system that can experience cavitation micro-pitting while in service. The content of the present paper consists of two distinct but interrelated parts. The first part aims to highlight that the use of computational fluid dynamics (CFD) simulations can constitute a potential tool for the prediction of cavitation erosion areas in scroll expander systems. For this purpose, a three-dimensional CFD, steady-state numerical simulation of the refrigerant working fluid is employed. Numerical results revealed the critical areas where cavitation bubbles are formed. These numerical critical areas are in direct qualitative agreement with the actual eroded regions by cavitation, which were found by microscopic observations across the steel plate on an after use, scroll expander system. The second part of the paper aims to further investigate the behaviour and the durability of the steel plate of the studied scroll expander system subjected to cavitation erosion by using an ultrasonic experimental test rig. Scanning electron microscopy and optical interferometer micrographs of the damaged surfaces were observed, showing the nature of the cavitation erosion mechanism and the morphological alterations of the steel plate samples. Experimental results are explained in terms of the cavitation erosion rates, roughness profile, accumulated strain energy, and hardness of the matrix. The experimental study can serve as a valuable input for future development of a CFD numerical model that predicts both cavitation bubbles formation as well as cavitation damage induced by the bubbles that implode on the steels plates.

Keywords: CFD, multiphase flow, ultrasonic cavitation, erosion, refrigerant, steel.
\end{abstract}

\section{INTRODUCTION}

Cavitation is a dynamic phenomenon known as a repeated formation and violent collapse of cavitation bubbles in a liquid caused by the instantaneous transition of liquid phase to gas phase due to rapid fluctuations of local pressure and is likely to occur in engineering applications such as diesel engine injectors, centrifugal pumps, etc. [1,2]. In contrast, in applications like scroll expander systems where pressure fluctuations are at critical levels and the refrigerant working fluid is in a vapour phase, cavitation can be initiated by the localised phase change [3]. Cavitation erosion damage is caused by material removal of a solid surface when it undergoes high-pressure impacts during the implosion of a single bubble or a cloud of bubbles. Implosion generates powerful micro-jets (with more than $1 \mathrm{GPa}$ hydrodynamic impact pressure) [4], large amplitude pressure waves (ten times greater than those at jet impact) [5] and secondary pressure waves (up to $0.5 \mathrm{GPa}$ ) [6], resulting to highly localised and transient surface stresses [7, 8]. Solid boundaries absorb that impact energy, leading to elastic deformation, plastic deformation and eventually to fracture [9].

In the present paper, a numerical and an experimental approach for the further understanding of the cavitation mechanism is used. The numerical study aims to identify the critical 
regions where cavitation can be generated by simulating the actual flow conditions of the refrigerant working fluid of the scroll expander system. The experimental study aims to study the evolution of the incubation pits into deep craters and to identify the durability of the scroll steel plate against cavitation attack.

It was found by field experiments that the critical region where cavitation can be initiated damaging the steel plate is in the first gas pocket of the scroll expander [3,10]. Thus, only this particular area of the scroll expander was taken into consideration for the numerical analysis. In a previous work by Tzanakis et al. [11], transient computational fluid dynamics (CFD) simulations of a simplified (2D) analogue of the scroll expander's first gas pocket indicated that due to the high inflow velocity of the gaseous working fluid (approximately $31 \mathrm{~m} / \mathrm{sec}$ from an inlet orifice diameter of $6 \mathrm{~mm}$ and a domain dimension along the inflow axis of $15 \mathrm{~mm}$ ), an instantaneous increase (within $5 \times 10^{-5} \mathrm{sec}$ after the inflow initiation) in the gas pressure (approximately 40 bar) is generated within the flow domain and particularly across the vicinity of the inlet nozzle where steel plates are located. This high pressure immediately drops to a much lower value (approximately 17 bar) within the first $0.02 \mathrm{sec}$ of the flow. This implies that as soon as the refrigerant gas phase enters into the flow domain, part of it is instantaneously transformed into liquid phase (according to its thermodynamic properties) due to the sudden pressure increase, and subsequently retransforms into gas phase producing cavitation bubbles due to the immediate pressure drop [11].

Therefore, in the current numerical simulation the liquid phase working fluid, which lasts for the very first $50 \mu \mathrm{sec}$ of the expansion process [11], is introduced into the computational domain at a high pressure (40 bars according to Tzanakis et al. [11]) and a multiphase numerical approach is used to identify the steady-state regions where high values (close to unity) of gas phase volume fractions are formed. These regions directly reveal the critical areas where cavitation bubbles are formed and indirectly the regions where cavitation damage is possible to be found. Interestingly, despite the overall assumptions in the numerical simulation, from a qualitative point of view, the results are in a very good agreement with the actual cavitation erosion damage areas from micro-pitting found across the steel plate of an after-part scroll expander system, after $1000 \mathrm{~h}$ of running time [10-12].

After the completion of numerical simulations, which clearly showed that cavitation bubbles are likely to be generated across the steel plate component and damage its surface, an experimental study was conducted. This is based on previous cavitation and bubble dynamics research outcomes [13], in order to investigate the durability of the scroll steel plate against cavitation attack and the formation of incubation pits [14] into deep craters. For this purpose, an ultrasonic transducer is utilised to produce cavitation bubbles. The resistance of the scroll's steel plate against cavitation erosion for a prolonged period of time is determined. Steel plate demonstrates a fatigue character induced by the level of the cavitation intensity while its endurance is significantly reduced after the first $5 \mathrm{~h}$ of exposure to cavitation impacts. Interestingly, correlations between the cavitation erosion rates, the roughness profile, the accumulated strain energy and the hardness of the matrix were found among the steel samples. This second part of the current work can serve as a valuable input for the future development of a CFDbased numerical model that predicts cavitation bubble formation as well as cavitation damage.

\section{NUMERICAL METHODOLOGY}

The numerical study aims to identify the critical regions where cavitation can be generated due to the expansion of the refrigerant working fluid. This occurs as the working fluid enters the first chamber of the scroll expander at a high velocity $31 \mathrm{~m} / \mathrm{sec}$ and violently impacts to 
the upper boundary wall. The simulated flow can be characterised as a multiphase flow of two immiscible fluids (vapour and liquid of the same fluid) that are separated by a clearly defined interface. The commercial CFD code ANSYS Fluent that is used for the simulations offers a wide range of multiphase models. The multiphase model that was adopted for the present simulation is the 'volume of fluid' (VOF) model. The VOF model is a surface tracking technique applied to a fixed Eulerian mesh. It is designed for two or more immiscible fluids, where the position of the interface between the fluids is of interest. In the VOF model, the fluids share a single set of momentum equations, and the volume fraction of each of the fluids in each computational cell is tracked throughout the domain. In other words, the flow within the first gas pocket of the scroll system that is investigated in the present paper is governed by the usual equations of mass (eqn (1)) and momentum conservation (eqn (2)), where $v$ is the velocity vector field, $p$ is the density, $\sigma$ is the stress tensor and $f$ is the body forces per unit volume

$$
\begin{gathered}
\frac{\partial v}{\partial t}+\nabla \cdot(p v)=0 \\
p\left[\frac{\partial v}{\partial t}+(v \cdot \nabla) v\right]=\nabla \sigma+f
\end{gathered}
$$

In more detail, a single set of momentum equations is solved for turbulent flow, using the finite volume method, and the tracking of the vapour-liquid interface throughout the flow domain is accomplished by the solution of a transport equation for the volume fraction $\alpha$ of the vapour phase (eqn (3))

$$
\frac{\partial a_{\mathrm{vapour}}}{\partial t}+\nabla\left(a_{\mathrm{vapour}} \cdot v\right)=0
$$

A major assumption is that in every control volume (computational cell), the sum of the volume fractions of vapour and liquid is equal to unity $\left(\alpha_{\text {vapour }}+\alpha_{\text {liquid }}=1\right)$. In other words, the volume fraction equation is not solved for the primary flow phase (in this case, refrigerant liquid); the primary phase volume fraction is calculated from the volume fraction of the secondary flow phase (in this case, refrigerant vapour) using the above assumption. The material properties appeared in all the above equations are determined by the presence of the component phases in each control volume as follows:

$$
\varphi=a_{\text {vapour }} \varphi+\left(1-a_{\text {vapour }}\right) \varphi_{\text {liquid }}
$$

where $\varphi_{\text {vapour }}$ and $\varphi_{\text {liquid }}$ are the vapour and liquid material properties of the working fluid (density, viscosity, etc.). In eqn (3), an additional source term for the vapour phase production is incorporated using the 'Zwart-Gerber-Belamri' Cavitation Model from the available cavitation models in ANSYSFluent 14.0. Finally, for turbulence closure, the Re normalisation-group (RNG) $k-\varepsilon$ model is applied.

Its general transport equations for the turbulence kinetic energy $k$ and the turbulence dissipation rate $\varepsilon$ can be described by the eqns (5) and (6), respectively:

$$
\frac{\partial}{\partial t}(\rho k)+\frac{\partial}{\partial x_{i}}\left(\rho k u_{i}\right)=\frac{\partial}{\partial x_{i}}\left(a_{k} \mu_{e f f} \frac{\partial k}{\partial x_{j}}\right)+G_{k}+G_{b}-\rho \varepsilon
$$




$$
\frac{\partial}{\partial t}(\rho \varepsilon)+\frac{\partial}{\partial x_{i}}\left(\rho \varepsilon u_{i}\right)=\frac{\rho}{\partial x_{j}}\left(a_{\varepsilon} \mu_{e f f} \frac{\partial \varepsilon}{d x_{j}}\right)+C_{1 \varepsilon} \frac{\varepsilon}{k}\left(G_{k}+C_{3 \varepsilon} G_{b}\right)-C_{2 \varepsilon} \rho \frac{\varepsilon^{2}}{k}-R \varepsilon
$$

where $u$ represents velocity, $\rho$ is the local mixture density, $G_{k}$ is the generation of turbulence kinetic energy due to mean velocity gradients, $G_{b}$ is the generation of turbulence kinetic energy due to buoyancy, $\alpha_{k}$ and $\alpha_{\varepsilon}$ are the inverse effective Prandtl numbers for $k$ and $\varepsilon$, respectively, $\mu_{\text {eff }}$ is the effective viscosity and $C_{1 \varepsilon}, C_{2 \varepsilon}$ and $C_{3 \varepsilon}$ are turbulence model constants. The term $R_{\varepsilon}$ in the $\varepsilon$ equation accounts for the effects of rapid strain and streamline curvature. Further details regarding all the above equations can be found in ANSYS Fluent Theory Guide [15].

\section{EXPERIMENTAL METHODOLOGY}

Cavitation erosion tests were utilised to analyse and evaluate the behaviour and durability of the scroll's steel plate against cavitation. The experiments were carried out based upon the modified ASTM G32-03 standard method of Tzanakis et al. [13]. An ultrasonic vibratory transducer with vibratory frequency of $20 \mathrm{kHz}$ and peak-to-peak vibratory amplitude of $50 \mu \mathrm{m}$ was deployed (Fig. 1). The high-intensity ultrasound was applied to the liquid medium generating cavitation bubbles. The liquid medium, used for cavitation tests, was distilled water with physical properties the same as in [13].

An optical emission spectrometer was used in distinguishing the scroll's steel plate in its individual chemical elements (Fig. 1). Steel plate was found to be a chromium alloy steel UNS G50986 (equivalent to AISI 50100) with measured hardness at $530 \pm 30 \mathrm{HV}$ and estimated yield strength $>415 \mathrm{MPa}$. Consequently, the scroll plate is machined into appropriate samples with dimensions of $15 \times 10 \times 0.3 \mathrm{~mm}$, which were shaped by a cutting machine. Then, the steel samples were implemented on a Bakelite base and polished in a few microns accuracy prior to the test $\left(\mathrm{Ra}=0.015 \pm 0.01 \mu \mathrm{m}\right.$ for a sample area of $\left.75 \mathrm{~mm}^{2}\right)$. The steel samples were mounted on the bottom of a 5-L experimental tank, while the tip of the horn is submerged into distilled water to a distance of $0.5 \mathrm{~mm}$ from the top of the steel sample. All the experiments were conducted in room temperature environment with the temperature of the distilled water at $23^{\circ} \mathrm{C}$. Additionally, after the completion of the cavitation tests, micro-hardness tests were performed using an 8.8-N applied load.

Cavitation erosion tests were performed over a prolonged period of time ranging from 1 to $8 \mathrm{~h}$. After the end of each experiment, the durability and resistance of the steel samples to

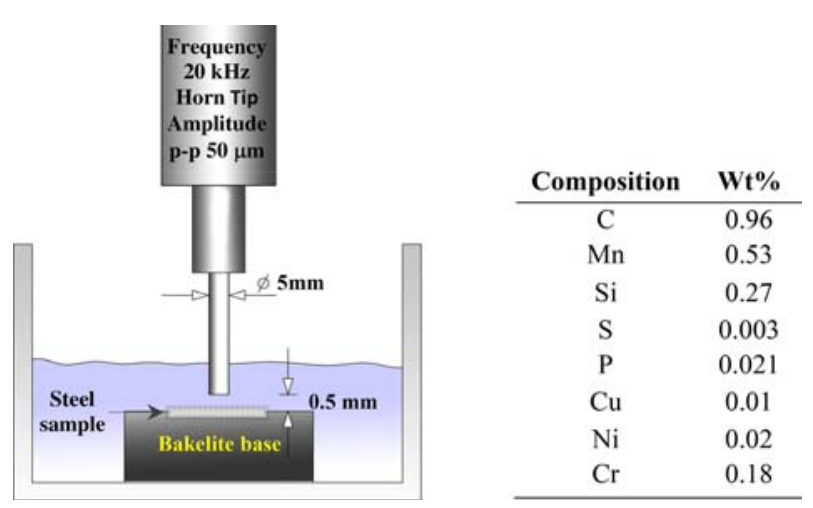

Figure 1: Cavitation experimental test rig and chemical analysis of the scroll's steel plate. 
cavitation was evaluated by measuring the erosion wear rates using profilometric techniques. The correlation between the strain energy, the roughness and the hardness evolution of the steel samples against their cavitation erosion rates was determined.

\section{NUMERICAL MODEL}

\subsection{Numerical model application}

The computational geometry and mesh that were constructed for the numerical simulation as well as the applied boundary conditions are illustrated in Fig. 2. As it can be seen, the computational domain (flow field) represents the first (inner) gas pocket of the examined scroll system when the rotating part is at its initial position. The main assumption is that the end boundaries of the first gas pocket are assumed to be open-flow boundaries with a prescribed pressure equal to the measured working pressure of the real system using laboratory equipment (pressure outlet boundary condition). This is mainly done for convergence purposes of the continuity equation, since a steady-state simulation was performed, aiming to identify what happens in the first gas pocket, from a qualitative point of view. At the inflow boundary, a 'velocity inlet' boundary condition was applied, where the velocity magnitude normal to the proposed boundary as well as the turbulence intensity and hydraulic diameter were prescribed. Another major assumption, as it was already discussed in the Introduction section, is that the liquid phase of the refrigerant enters the computational domain from the inlet port. Finally, the solid boundaries of the domain were defined as stationary 'walls' with a no-slip boundary condition. The actual dimensions of each scroll are depicted in Fig. 3.

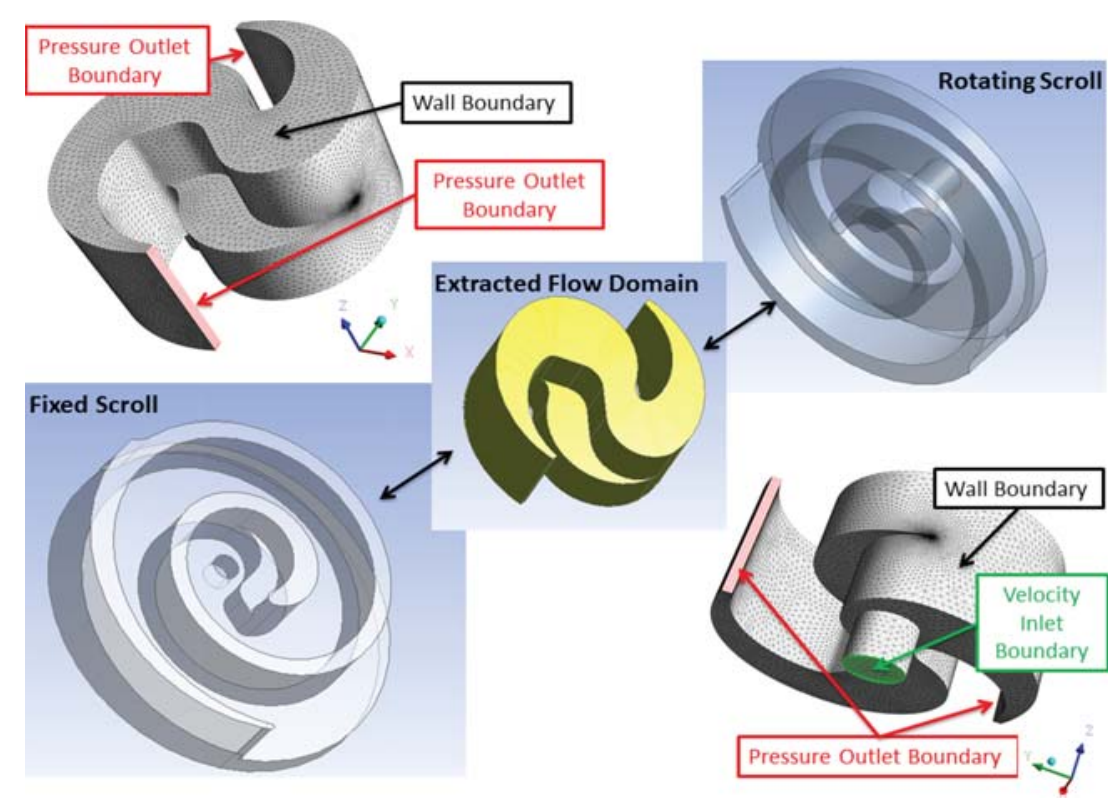

Figure 2: Geometry, computational mesh and boundary conditions of the first expansion pocket of the scroll expander. 

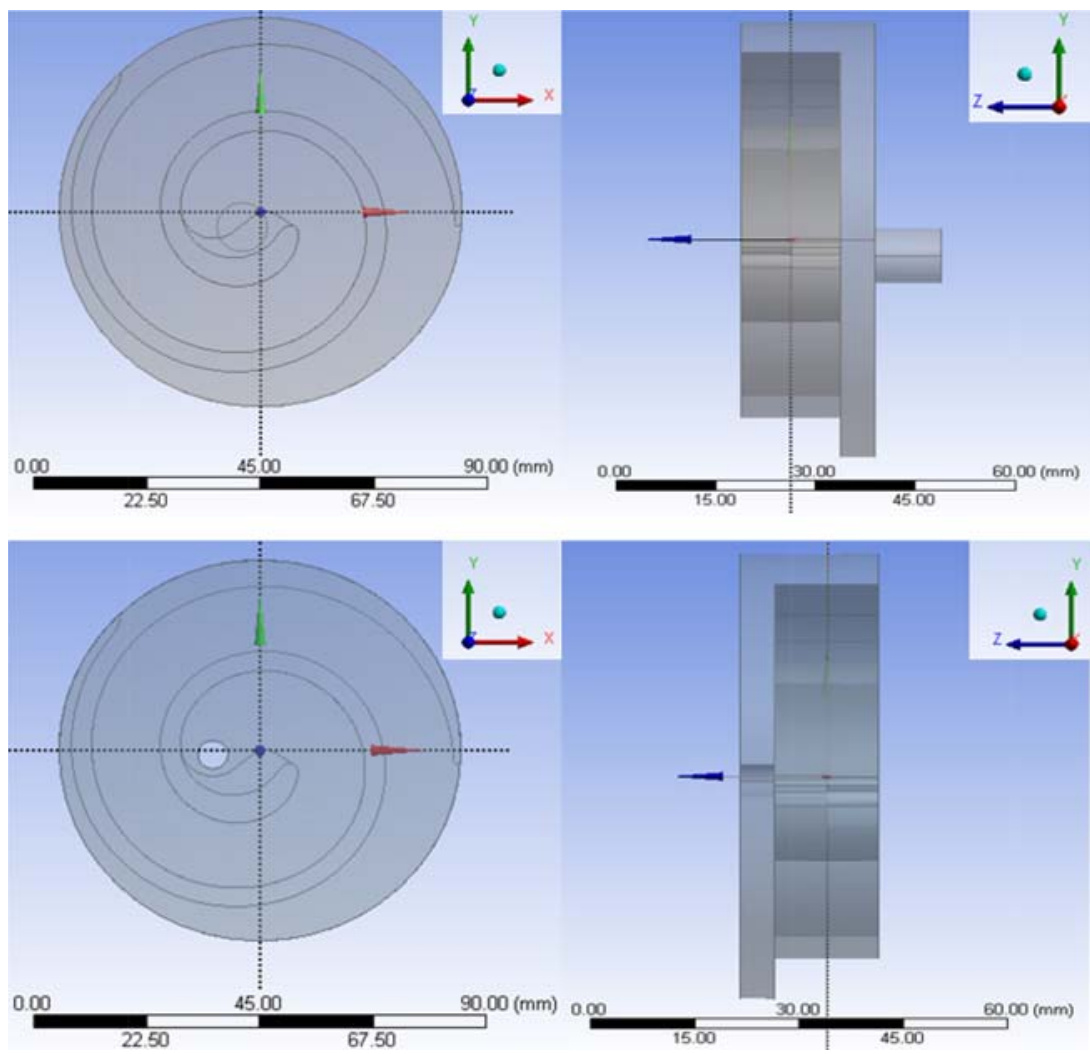

Figure 3: Top and side views of the upper (top of figure) and lower (bottom of figure) scroll geometries that were used for the extraction of the simulation domain.

At this point, it should be mentioned that due to scroll's complicated design and its geometrical restrictions, a 3D reconstruction model using CAD methods was not feasible. Thus, a laser scanning facility was used to generate a detailed, high-resolution 3D model of the actual scroll expander system with minimal surface noise. Laser facility uses advanced non-contact laser sensors to quickly generate precise models with very high scanning resolutions (up to $0.2 \mathrm{~mm}$ ). The 3D model in turn was introduced into the computational domain of ANSYS and used for the numerical analysis.

A total number of 526,630 computational cells (control volumes) were used for the spatial discretisation of the computational domain. The solution was also verified using finer and coarser meshes, indicating negligible differences in the results. Thus, the solution that is presented in the present paper can be considered to be mesh independent. The majority of the computational domain consists of 'tetrahedral' cells with a typical edge size of $75 \times 10^{-5} \mathrm{~m}$ that are locally refined in regions where increased computational accuracy was required. In the vicinity of the solid wall boundaries of the domain, the computational mesh has been inflated by five parallel layers of prismatic cells in order to better account for flow quantities at the near-wall regions. The first layer height from the bottom wall is $0.0001 \mathrm{~m}$, whereas the height of the rest four layers progressively increases with a factor $n=1.2$, away from the solid boundaries. The overall conditions that were used in order to initialise the flow domain were 
Table 1: Numerical simulation initial conditions (measured in laboratory experiments).

\begin{tabular}{lc}
\hline Flow initial conditions & \\
\hline Velocity inlet & \\
$\quad$ Velocity magnitude $(\mathrm{m} / \mathrm{sec})$ & 31 \\
Pressure outlets & \\
$\quad$ Pressure (bar) & 12 \\
Interior domain & \\
$\quad$ Pressure (bar) & 40 \\
Fluid material properties & \\
Refrigerant vapour $\left(\right.$ at $\left.150^{\circ} \mathrm{C}\right)$ & 47.377 \\
$\quad$ Density $\left(\mathrm{kg} / \mathrm{m}^{3}\right)$ & $1.1115 \times 10^{-5}$ \\
$\quad$ Dynamic viscosity $(\mathrm{kg} / \mathrm{m} \mathrm{s})$ & \\
Refrigerant liquid $\left(\right.$ at $\left.150^{\circ} \mathrm{C}\right)$ & 460.08 \\
$\quad$ Density $\left(\mathrm{kg} / \mathrm{m}^{3}\right)$ & $7.5931 \times 10^{-5}$ \\
$\quad$ Dynamic viscosity $(\mathrm{kg} / \mathrm{m} \mathrm{s})$ & \\
\hline
\end{tabular}

taken from laboratory measurements of the actual scroll expander systems in the work done by Tzanakis [16] and summarised in Table 1. Finally, the basic characteristics and parameters of the optimum (final) model set-up within ANSYS Fluent are summarised in Table 2.

\subsection{Numerical model results}

To visualise and better understand the calculated flow field in the interior domain, 3D streamlines (Fig. 4a) as well as 2D velocity vector fields at two vertical planes perpendicular to each other (Fig. 4b and c) are depicted in Fig. 4. As it can be seen, the resulting refrigerant flow within the gas pocket is quite complex presenting various eddies and velocity magnitude fluctuations. This flow-field complexity seems to be attributed to the highly turbulent nature of the flow as well as to the complexity of the gas pocket geometric boundaries resulting to pressure fluctuations and generation of bubbles in particular regions across the steel plate.

Specifically, the numerical model predicted the critical regions of the steel plate where cavitation can be generated, indirectly revealing the corresponding high volume fraction regions (close to unity). This generated vapour phase is plotted on the bottom wall boundary (steel plate) of the computational domain. These regions can be found in Fig. 5a, where the volume fraction contours of the refrigerant's vapour phase are illustrated on the bottom boundary of the computational domain. As it can be seen, high volume fraction areas on the bottom boundary are found in two major regions: region 1 where it is bounded between the inlet boundary and the inner vertical wall boundary of the fixed scroll lobe and region 2 where it forms a narrow zone parallel to the outer vertical wall of the fixed scroll lobe, starting just downstream of the lobe tip and extending up to one end of the first gas pocket. Additionally, to visualise the numerically predicted vapour phase regions within the computational domain, in Fig. 5c and d, the refrigerant's vapour volume fraction contours are plotted on the same vertical planes as the ones used in Fig. $4 \mathrm{~b}$ and c, respectively.

Interestingly, these numerically predicted critical cavitation regions are in direct qualitative agreement with the actual cavitation erosion damage areas by micro-pitting found across 
Table 2: Model set-up parameters and characteristics in ANSYS Fluent.

\begin{tabular}{ll} 
A. Model set-up & \\
\hline A1. Solver & \\
Type & Pressure-based \\
Velocity formulation & Absolute \\
Space & 3D \\
Time & Steady \\
A2. Multiphase model & \\
Name & Volume of fluid (VOF) \\
Scheme & Implicit \\
No. of phases & 2 \\
A3. Turbulence model & \\
Name & Realisable $k-\varepsilon$ \\
Near-wall treatment & Standard wall functions \\
A4. Cavitation model & \\
$\quad$ Name & Zwart-Gerber-Belamri \\
Vaporisation pressure (bar) & 15.9 (at $\left.150^{\circ} \mathrm{C}\right)$ \\
A5. Phases & \\
$\quad$ Primary & Refrigerant liquid \\
Secondary & Refrigerant vapour \\
\hline B. Solution & \\
\hline B1. Methods & \\
Pressure-velocity coupling scheme & PISO \\
Spatial discretisation schemes & \\
Gradient & Least-squares cell based \\
Pressure & PRESTO \\
Momentum & Second-order upwind \\
Turbulent kinetic energy & Second-order upwind \\
Turbulent dissipation rate & \\
\hline
\end{tabular}

the steel plate of an after-part scroll expander system, after $1000 \mathrm{~h}$ of running time, in the experimental work of Tzanakis et al. [10-12] (Fig. 5b). In the proposed field experiments, the actual scroll configuration of the numerical study has been used, with the same refrigerant flow conditions and characteristics.

\section{EXPERIMENTAL RESULTS}

\subsection{Microscopic examination}

After the completion of the numerical analysis and the outcomes showing that cavitation bubbles can be definitely generated and consequently damage the steel plate component of the scroll expander system, the next step was to evaluate the behaviour and durability of the 


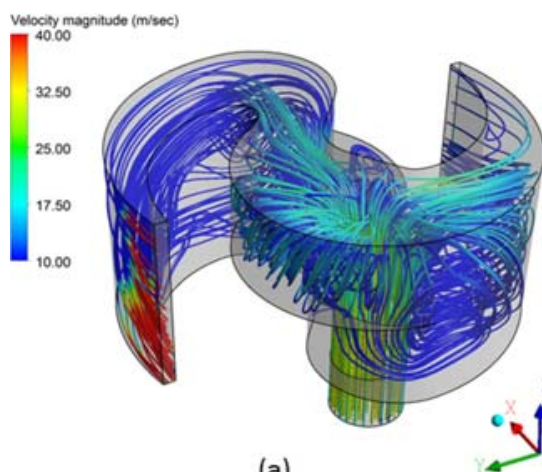

(a)

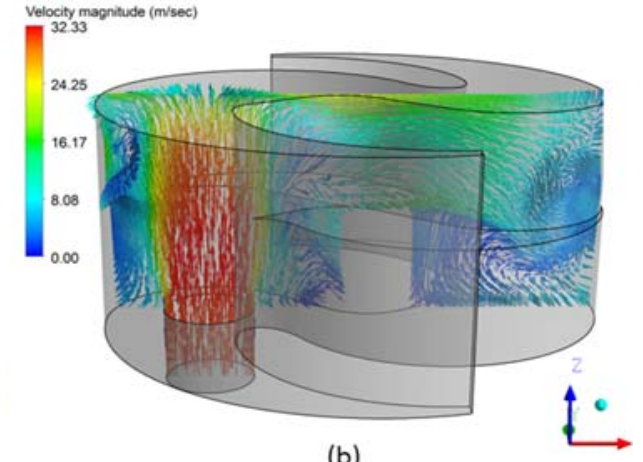

(b)

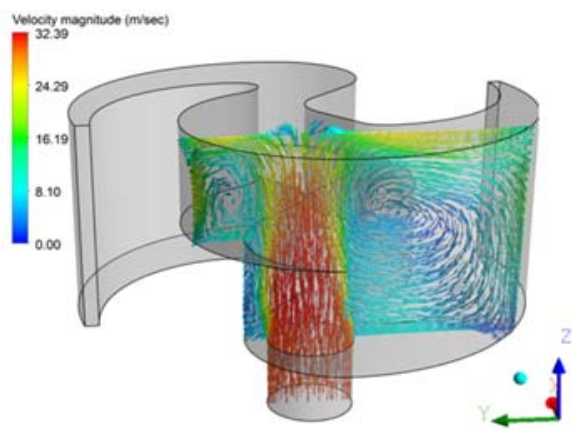

(c)

Figure 4: Flowfield visualisation: (a) 3D streamlines coloured by velocity. Velocity vector field in a vertical (b) ZX plane and (c) ZY plane normal to the inflow boundary coloured by velocity.

steel plate against cavitation attack. Ultrasonic cavitation tests were conducted for different time periods starting from $1 \mathrm{~h}$ up to $8 \mathrm{~h}$. The incubation pits, which were formed in the early stages of the cavitation mechanism, characterised as micro-pitting, can significantly grow by linking, including cracks and grooves, general roughening of the surface $[14,17,18]$. The enlargement of the pits is accompanied by additional volume loss accelerating the erosion rate of the materials. The mechanism of pit growth leads to the formation of deep and wide craters penetrating the sample's surface. These areas were named 'ring areas' because of the shape they obtain after the end of the ultrasonic cavitation process.

A series of SEM micrographs in Fig. 6 shows the eroded surface of the scroll's steel plate at different stages of the cavitation process. The images were taken in each of the selected test intervals to get an overview of the attenuation of the pits and their formation into craters. After $1 \mathrm{~h}$ of testing, the initial stage of cavitation erosion is shown as pits formation dominates the erosion regime (Fig. 6a). The next stage is after $3 \mathrm{~h}$ of exposure where a crater shape is formed (Fig. 6b). The periphery of the crater is clearly visible. Its core consists of pits, which show increased density and size. After $5 \mathrm{~h}$ of testing, cavitation damage is spread to the vicinity areas of the 'ring' and significantly erosion damage is observed (Fig. 6c). However, no significant material loss was noticed as erosion restricted within the upper substrate of the steel surface, while the crater depth remained to similar levels as in Fig. 6b. Finally, after $8 \mathrm{~h}$ of testing, the crater was severely eroded forming a deep valley. The edges of the periphery 


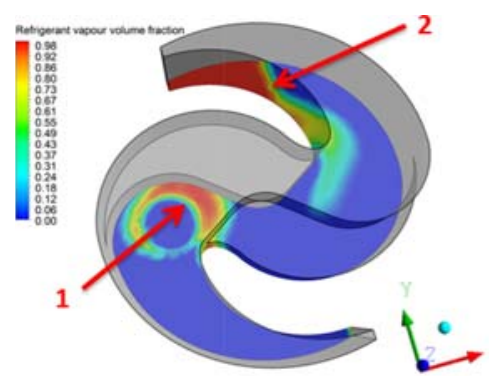

(a)

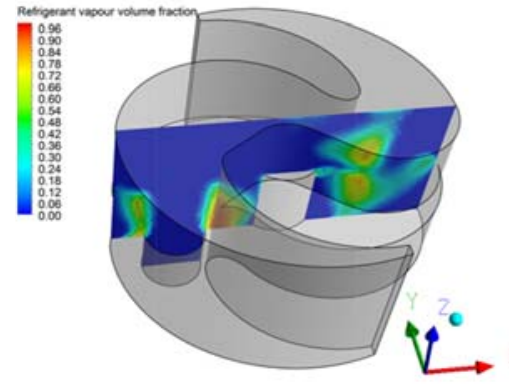

(c)

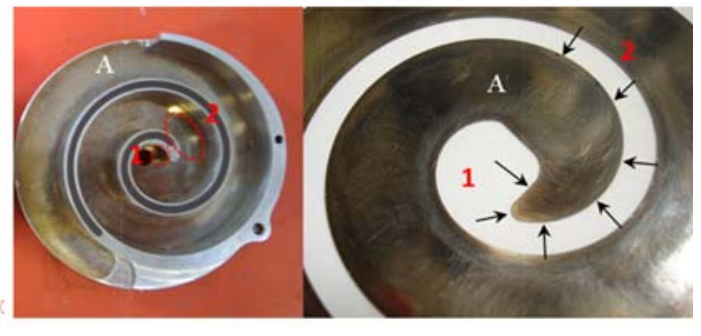

(b)

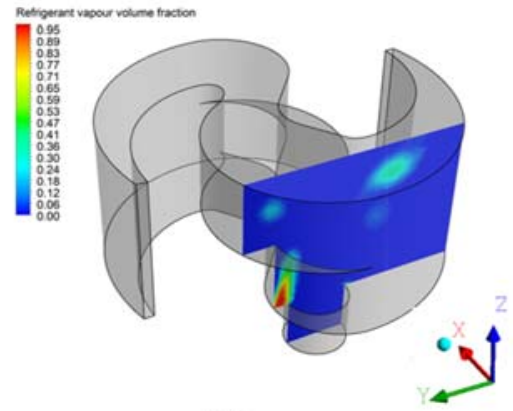

(d)

Figure 5: (a) High vapour volume fraction regions showing the critical regions susceptible to cavitation. (b) Actual cavitation regions identified by optical microscope. Volume fraction contours in a vertical (c) ZX plane and (d) ZY plane normal to the inflow boundary.

were very sharp and sloppy while significant loss of material was noticed. Closer observation demonstrated the increased depth of the crater (Fig. 6d, white arrow).

\subsection{Erosion rates}

Measurements were carried out to monitor the volume loss, the roughness profile and the mean depth erosion rate (MDER) of the steel samples over the different stages of the cavitation erosion tests.

The erosion loss of material was expressed in terms of the MDER (eqn (7)) and the roughness in terms of $R_{a}$, which is the most common roughness parameter.

Both of the parameters were defined as it follows:

$$
\begin{gathered}
\operatorname{MDER}(\mu \mathrm{m} / \mathrm{h})=10 \frac{\Delta W}{\rho A \Delta t} \\
R_{a}=\frac{1}{L} \int_{0}^{L}|z(x)| d x
\end{gathered}
$$

where $\Delta W$ is the weight loss in $\mathrm{mg}, \Delta t$ is the test time in hours, $A$ is the cavitated area of the specimen in $\mathrm{cm}^{2}, \rho$ is the density of the specimen in $\mathrm{g} / \mathrm{cm}^{3}, \mathrm{~L}$ is the length area of the sample surface in $\mu \mathrm{m}$ and $\mathrm{z}$ is the average value of the asperities height in $\mu \mathrm{m}$.

According to Fig. 7, all of the parameters are proportionally increasing with time. Specifically, up to $5 \mathrm{~h}$ exposure to cavitation, steel plate exhibits low volume loss $\left(<0.01 \mathrm{~mm}^{3}\right)$ 

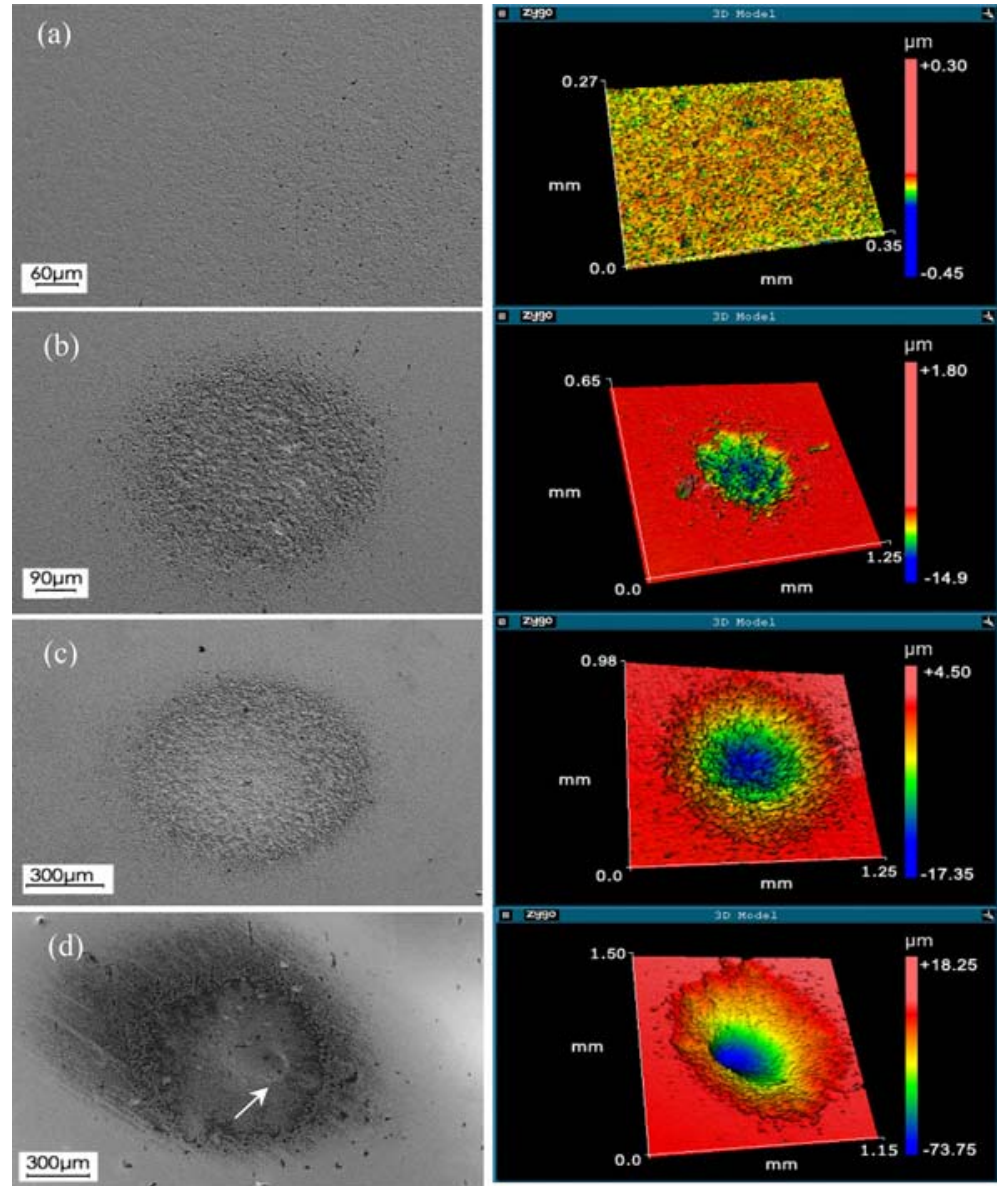

Figure 6: A series of SEM and ZYGO interferometer micrographs showing the evolution of cavitation pits into crater at different stages: (a) $1 \mathrm{~h}$, (b) $3 \mathrm{~h}$, (c) $5 \mathrm{~h}$ and (d) $8 \mathrm{~h}$.

and MDER (around $0.1 \mu \mathrm{m} / \mathrm{h}$ ) while roughness is changed with a relative low rate. Then, its endurance was dramatically reduced and the erosion rate was steeply increased (more than $0.4 \mu \mathrm{m} / \mathrm{h}$ ) while its volume loss increased by five times and its roughness profile by four times. The heat influence, the repeated impact pressure and the interactions between the dislocations and the grain structure cause the material flow and to significantly drop its hardness (Fig. 8) made it vulnerable to cavitation. Hence, over a prolonged period $(8 \mathrm{~h})$ of exposure to cavitation impacts, the steel plate of the scroll faced inevitable severe damage.

\subsection{Hardness}

The change in hardness can be closely correlated with the erosion resistance (REC) of the steel samples. Generally, higher resistance implies higher hardness values. The cavitation 

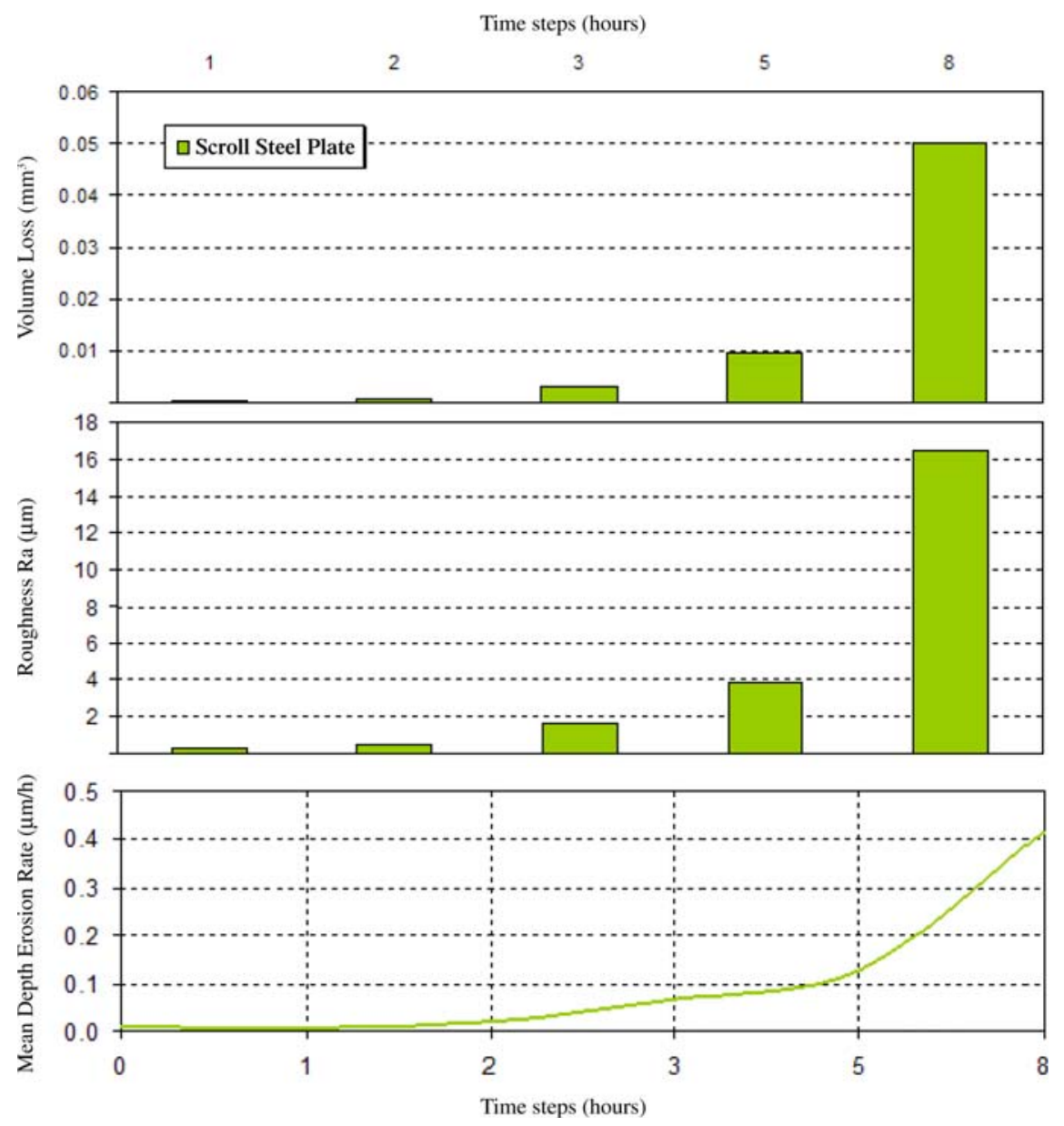

Figure 7: Variation of the volume loss, roughness profile and MDER of the scroll's steel plate for different time steps.

erosion resistance (REC) is defined as the reciprocal of the mean erosion rate and is expressed by the following equation:

$$
\operatorname{REC}(\mathrm{h} / \mu \mathrm{m})=\frac{1}{\mathrm{MDER}}
$$

In every steel sample, the hardness alterations depend on the intention and the time duration of the cavitation impacts. If conditions are suitable, work hardening on the steel samples occurs, otherwise rapid damage can occur.

Figure 8 shows the hardness monotonically increasing with the increment of the erosion resistance. Interestingly, for the test duration of $2 \mathrm{~h}$, the hardness of the steel has slightly increased. This can be interpreted by the fact that dislocation movements were possibly restricted, increasing their density in those areas. Consequently, the hardness and the brittleness of the steel were increased making it vulnerable to cavitation. However, after $3 \mathrm{~h}$ of test, 


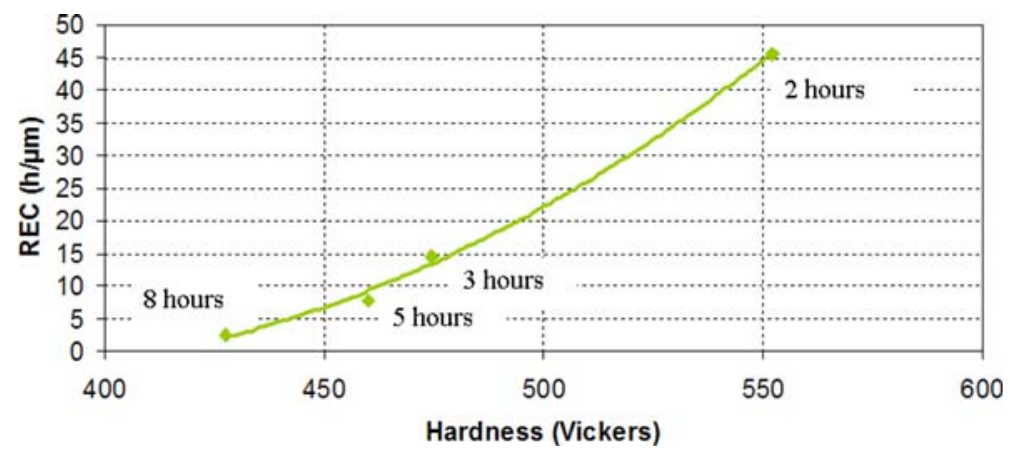

Figure 8: Hardness against erosion resistance of the steel plate for different time steps.

a rapid decrease in the hardness value of about $100 \mathrm{HV}$ is observed implying the rapid reduction of its erosion resistance. From that point onwards, the high carbon steel plate shows its brittle character, incapable of resisting the massive striking of the micro-jets, lowering its hardness values significantly.

\subsection{Strain energy}

The cavitation erosion mechanism has a fatigue character; hence, the damage needs time to be developed. Since the erosion damage is caused by consecutive impacts similar to a fatigue phenomenon, the accumulated strain energy can indicate the critical point where the damage becomes crucial for the steel. Strain energy estimates the rate of the erosion and the resistance of the samples against cavitation. Thus, the accumulated strain energy can be expressed by the following equation:

$$
\sum E n=V \sum\left(\frac{\sigma^{2}}{2 E}\right)
$$

where $V$ is the volume loss in mg, $\sigma$ is the yield strength in MPa and $E$ is the Young modulus in GPa.

The dependency of the accumulated strain energy as a function of the crater depth of the steel samples is depicted in Fig. 9. As shown, there are three stages of the erosion evolution according to the strain energy results. The first stage is the inception of cavitation pits until the formation of small craters. In this stage, strain energy slightly increases for the first $3 \mathrm{~h}$ of the test. Then, for the next $2 \mathrm{~h}$, the crater depth stabilises to a value around $20 \mu \mu \mathrm{m}$ as volume loss is mainly observed from the peripheral areas of the crater rather than at places within the crater (Fig. 6c). Consequently, strain energy rate, which is accumulated in a larger area within the mass of the steel, increases by about three times. When the strain energy exceeds the limit of the $15 \mathrm{~mJ}$, the eroded damage is localised forming deep craters (Fig. 6d). In that stage, a notable volume loss is observed while the erosion rates are significantly accelerated.

\section{SUMMARY AND CONCLUSIONS}

The cavitation mechanism within the first gas pocket of a scroll expander system is assessed by applying multiphase numerical methods. A three-dimensional scroll model is validated and compared with field experimental measurements from the expander unit. The performance of 


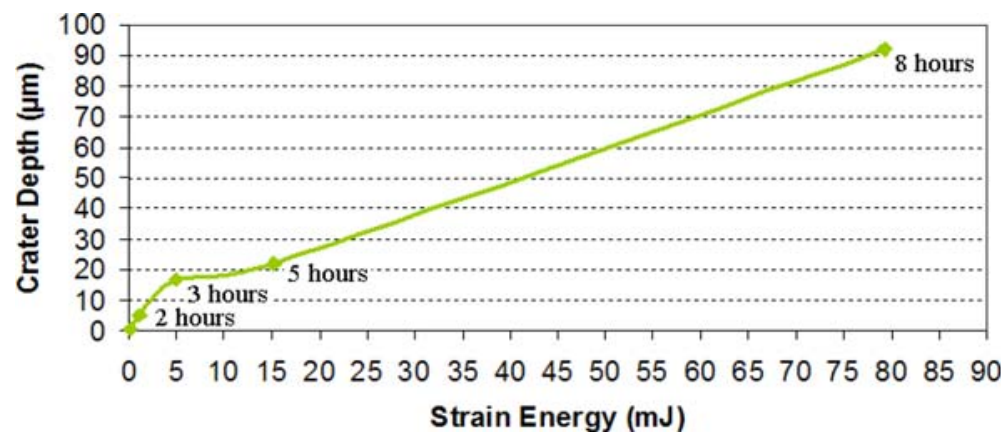

Figure 9: Correlation between the accumulated strain energy and the crater depth of the steel plate for different time steps.

the scroll's steel plate in relation to cavitation erosion behaviour is derived by the application of laboratory ultrasonic techniques. The main findings are listed below:

1. Numerical results revealed the critical areas where cavitation is likely to happen. Interestingly, these results are in direct qualitative agreement with the actual eroded regions by cavitation micro-pitting, which were found across the steel plate of an after use scroll expander system.

2. The most critical regions of the steel plate susceptible to cavitation erosion are found between the inlet boundary and the inner vertical wall boundary of the fixed scroll lobe as well as in a narrow zone parallel to the outer vertical wall of the fixed scroll lobe, starting just downstream of the lobe tip and extending up to one end of the first gas pocket.

3. Experimental results from the ultrasonic transducer determine the endurance of the scroll's steel plate material against cavitation attack. Steel plate exhibits a good performance against cavitation for the first $5 \mathrm{~h}$ of the test, according to the volume loss and the cavitation erosion rate graphs. After that time step, repeated impacts of cavitation pulses lead to accelerative breakage of the substrate as its crystal structure cannot sustain the new energy income and the material experiences severe erosion.

4. The evolution of the roughness profile with time is determined. It is characteristic that roughness steadily increases until the first $5 \mathrm{~h}$ of exposure to cavitation with a relatively low rate, while after that point the proposed rate shows a rapid increment by approximately four times accelerating the erosion rate of the steel samples.

5. Cavitation-erosion mechanisms affecting the durability of the scroll expander's steel plate explicitly depend on its hardness and the ability of the material to accumulate strain energy. Hardness is proved to be one of the key factors in increasing the lifetime of the steel plate as it is directly correlated with the erosion resistance of the material. Erosion damage was localised when accumulated strain energy of more than $15 \mathrm{~mJ}$ is applied to the specimen by repeated impact.

For future investigation, it is necessary to further extend the content of the present investigation, conducting experimental measurements among similar industrial steel grades, aiming to identify a higher cavitation-resistant steel material. Additionally, a transient numerical application of the proposed scroll expander system needs to be conducted, minimising the overall assumptions by incorporating the actual movement of the rotating scroll as well as the 
instantaneous phase change of the inflowing vapour phase into liquid and vice versa. Nevertheless, from the overall outcomes of the present paper, it is evident that carefully designed and tested CFD cavitation models in conjunction with modern experimental measuring techniques can serve as a quite attractive and valuable tool for the evaluation and prediction of cavitation damage in scroll expander systems or similar automotive turbine units.

\section{REFERENCES}

[1] Andriotis, A., Gavaises, M. \& Arcoumanis, C., Vortex flow and cavitation in diesel. Journal of Fluid Mechanics, 610, pp. 195-215, 2008. doi: http://dx.doi.org/10.1017/ $\underline{\text { S0022112008002668 }}$

[2] Nemdili, A., Experimental study of the influence of geometrical parameters on the cavitation of a small centrifugal pump. WIT Transactions on Engineering Sciences, 84, pp. 89-97, 2005.

[3] Tzanakis, I., Hadfield, M. \& Khan, Z., Durability of domestic scroll compressor systems. WIT Transactions on Engineering Sciences, 62, pp. 229-240, 2009. doi: http:// dx.doi.org/10.2495/SECM090211

[4] Tzanakis, I., Eskin, D.G., Georgoulas, A. \& Fytanides, D.K., Incubation pit analysis and calculation of the hydrodynamic impact pressure from the implosion of an acoustic cavitation bubble. Ultrasonics Sonochemistry, 21, pp. 866-878, 2014 . doi: http:// dx.doi.org/10.1016/j.ultsonch.2013.10.003

[5] Tong, R.P., Schiffers, W.P., Shaw, S.J., Blake, J.R. \& Emmony, D.C., The role of 'splashing' in the collapse of a laser-generated cavity near a rigid boundary. Journal of Fluid Mechanics, 380, p. 339, 1999. doi: http://dx.doi.org/10.1017/S0022112098003589

[6] Brujan, E.A., Ikeda, T. \& Matsumoto, Y., Shock wave emission from a cloud of bubbles. Soft Matter, 21, pp. 5777-5783, 2012. doi: http://dx.doi.org/10.1039/c2sm25379h

[7] Krella, A. \& Czyzniewski, A., Influence of the substrate hardness on the cavitation erosion resistance of TiN coating. Wear, 263, pp. 395-401, 2007. doi: http://dx.doi. org/10.1016/j.wear.2007.02.003

[8] Williams, P.R., Williams, P.M. \& Brown, S.W.J., A technique for studying liquid jets by cavitation bubble collapse under shockwaves, near a free surface. Journal of NonNewtonian Fluid Mechanics, 72, pp. 101-110, 1997. doi: http://dx.doi.org/10.1016/ S0377-0257(97)00020-7

[9] Mann, B.S., High-energy particle impact wear resistance of hard coatings and their application in hydroturbines. Wear, 237, pp. 140-146, 2000. doi: http://dx.doi. org/10.1016/S0043-1648(99)00310-5

[10] Tzanakis, I., Hadfield, M., Thomas, B., Noya, S.M., Austen, S. \& Henshaw, I., Future perspectives of sustainable tribology. Renewable and Sustainable Energy Reviews, 16(6), pp. 4126-4140, 2012. doi: http://dx.doi.org/10.1016/j.rser.2012.02.064

[11] Tzanakis, I., Hadfield, M., Georgoulas, A. \& Kotsovinos, N., Cavitation damage observations within scroll expander lubrication systems. WIT Transactions on Engineering Sciences, 66, pp. 261-272, 2010. doi: http://dx.doi.org/10.2495/TD100221

[12] Tzanakis, I. \& Eskin, D.G., Cavitation erosion behaviour of martensitic steels with different carbon content using ultrasonic methodology. Materials Science and Engineering: A (Submitted).

[13] Tzanakis, I., Hadfield, M. \& Henshaw, I., Observations of acoustically generated cavitation bubbles within typical fluids applied to a scroll expander lubrication system. $E x$ perimental Thermal and Fluid Science, 35(8), pp. 1544-1554, 2011. doi: http://dx.doi. org/10.1016/j.expthermflusci.2011.07.005 
[14] Tzanakis, I., Garland, N. \& Hadfield, M., Cavitation damage incubation with typical fluids applied to a scroll expander system. Tribology International, 44(12), pp. 16681678, 2011. doi: http://dx.doi.org/10.1016/j.triboint.2011.06.013

[15] ANSYS Fluent 14.5 ANSYS, Inc. Release 14.5, October 2012.

[16] Tzanakis, I., Sustainable design and durability of domestic micro combined heat and power scroll expander systems. Ph.D. Thesis, Bournemouth University, 2010.

[17] Karunamurthy, B., Hadfield, M., Vieillard, C. \& Morales G., Cavitation erosion in silicon nitride: experimental investigations on the mechanism of material degradation. Tribology International, 43, pp. 2251-2257, 2010. doi: http://dx.doi.org/10.1016/j.triboint.2010.06.012

[18] Asi, O., Failure of a diesel engine injector nozzle by cavitation damage. Engineering Failure Analysis, 13, pp. 1126-1133, 2006. doi: http://dx.doi.org/10.1016/j.engfailanal.2005.07.021 